\title{
Rotavirus vaccine withdrawal in the United States: The role of postmarketing surveillance
}

Gilles Delage MD MSc, Laboratoire de santé publique du Québec, Sainte-Anne-de-Bellevue, Québec

$\mathrm{O}$ August 31, 1998, Rotashield (Wyeth Laboratories, Marietta, Pennsylvania) was licensed by the United States Food and Drug Administration for oral administration to infants at two, four and six months of age in the United States. This product is a live, attenuated rhesus rotavirusbased tetravalent vaccine (RRV-TV). Each dose of vaccine contains $1 \times 10^{5}$ plaque forming units of each of four strains: one is a rhesus rotavirus strain with human serotype 3 specificity, and the three other strains are single gene human-rhesus reassortants that contain the human gene for serotype 1,2 and 4 antigens, respectively, along with other genes from the parent rhesus rotavirus strain. Shortly following licensure of the vaccine in the United States, the Advisory Committee on Immunization Practices (ACIP) (1) recommended routine immunization with three oral doses of RRV-TV for infants at age two, four and six months of age for the prevention of rotavirus disease in the United States. The rationale for recommending use of the vaccine was the following.

- The rate of illness due to rotavirus among children in the United States is quite high (2).

- Although the implementation of oral rehydration programs to prevent dehydration has reduced to a great extent mortality due to diarrhea-associated disease in the United States (3), rotavirus continues to cause significant morbidity particularly in terms of hospitalizations $(4,5)$.

- Trials of RRV-TV vaccine in the United States, Finland and Venezuela showed efficacy rates of approximately $80 \%$ for the prevention of severe disease and of $48 \%$ to $68 \%$ against all rotavirus induced diarrheal episodes (6-10).
- Prelicensure clinical trials data suggested that the vaccine was quite safe (1). Excess fever, decreased appetite, irritability and decreased activity were reported after the first dose of the vaccine. Only one study suggested an increased rate of diarrhea after the first dose of vaccine compared with placebo recipients (8). There was no statistically significant difference between vaccinees and placebo recipients in the rate of intussusception (five of 10,000 recipients of any reassortant vaccine versus one of 4632 placebo recipients) (1).

The American Academy of Pediatrics also recommended RRV-TV for use in infants two, four and six months of age for prevention of rotavirus disease. However, it stated that "routine implementation of this recommendation will require reconciliation of related economic issues" (11).

From September 1, 1998 to July 7, 1999, 15 cases of intussusception among infants who had received RRV-TV were reported to the Vaccine Adverse Event Reporting System (VAERS) (12). Of the 15 infants, 11 developed intussusception following the first of the three dose series and 12 developed the symptoms within one week of receiving any dose of RRV-TV. Intussusception was confirmed radiographically in all 15 patients. Eight infants required surgical reduction, and one required resection of $18 \mathrm{~cm}$ of the distal ileum and proximal colon. Histopathological examination of the distal ileum indicated a lymphoid hyperplasia and ischemic necrosis. All infants recovered. The median age of the patients was three months, and reports were received from seven states. Based on prevaccine data on the incidence of intussusception in the United States and based on vaccine distribution data, 14.7 cases of intussusception were to be expected within one week of receipt of rotavirus vaccine due to temporal associa- 
tion without causation. However, in light of under-reporting of vaccine-associated adverse effects, these data suggested an increased risk of intussusception following rotavirus vaccination. In addition, surveillance data from the Northern California Kaiser Permanente Health Maintenance Organization and from the states of Tennessee and Minnesota also showed a trend towards the increased risk of intussusception following receipt of rotavirus vaccine (12). Although none of these findings were found to be conclusive, the consistency of the findings from the three data sources raised strong concerns. Based on these concerns, the Centers for Disease Control and Prevention (Atlanta, Georgia) recommended postponing administration of RRV-TV to children scheduled to receive the vaccine at least until November 1999. The manufacturer in consultation with the Food and Drug Administration voluntarily ceased further distribution of the vaccine.

By the beginning of October 1999, 101 confirmed and presumed cases of intussusception had been reported to VAERS. The rate of reporting increased greatly after the July 1999 announcement of the temporary postponement of rotavirus vaccination. Fifty-two patients required surgery, nine required bowel resection and one patient died. Fifty-seven of these children developed intussusception within seven days of vaccine dose, and $70 \%$ developed their intussusception after the first dose. There was a clear clustering of cases from day 3 to day 8 after the dose of vaccine. In case-control studies, risk ratios of developing postvaccine intussusception varied from seven to 30 , depending on the study and type of analysis. It was estimated that these data would have translated into around 1200 potential additional cases of intussusception annually in the United States if rotavirus vaccine policy had been fully implemented. Based on these data, the ACIP concluded that intussusception frequency was significantly increased in the first one to two weeks after vaccination, particularly after the first dose, and decided to withdraw its rotavirus vaccine recommendations (13). The American Academy of Pediatrics also withdrew its recommendations. On October 15, 1999, WyethLederle Vaccines announced that it was withdrawing the Rotashield vaccine from the market and requested the immediate return of all distributed doses.

These events demonstrate very clearly that postmarketing surveillance is an essential component of vaccine program implementation. Data obtained before licensure did not reveal an excess risk of intussusception following vaccination with rotavirus vaccine. However, postlicensure surveillance showed quite convincingly that rotavirus vaccine administration was associated with an increased risk of intussusception, particularly in the week following administration of the first dose. These events also demonstrate the power of active surveillance systems: Preliminary data from active systems in Minnesota and the Northern California Kaiser Permanente Health Maintenance Organization suggested an increased risk of intussusception following RRV-TV. Further accumulation and analysis of the data both from the VAERS and from the various postmarketing surveillance systems led the expert committees to withdraw their recommendations concerning the use of this vaccine.
In Canada, the vaccine had not yet been approved for use by the Therapeutic Products Program when data suggesting that the vaccine might be associated with an increased risk of intussusception became known. This vaccine is not expected to be approved in Canada, at least in the foreseeable future. However, other vaccines have recently been approved for use, for example, varicella, and others should soon be approved for use (conjugate pneumococcal and meningococcal). The events surrounding the introduction of the rotavirus vaccine show clearly that Canada needs to have a strong postmarketing surveillance system in place, particularly for newly introduced vaccines.

What systems exist in Canada? The Immunization Monitoring Program ACTive (IMPACT), an active surveillance system for severe side effects following immunization, is a central component of the postmarketing surveillance system in Canada (14). This program is operated under contract by the Canadian Paediatric Society, with funds provided by Health Canada, for the primary purpose of postmarketing surveillance of vaccine safety. The surveillance system is based upon 11 pediatric referral centres (soon to be 12) extending from St John's, Newfoundland to Vancouver, British Columbia. These centres comprise over 2000 paediatric beds and admit over 90,000 children annually, encompassing about $80 \%$ of paediatric admissions to Canadian academic centres. The investigators are infectious diseases specialists who act as volunteers. Each centre employs a nurse monitor who carries out the routine active surveillance activities in each centre. In addition to IMPACT, Health Canada operates a passive surveillance system similar to VAERS in the United States, which collects data on adverse reactions following immunizations reported by physicians and public health units (15). Finally, Health Canada has set up the Advisory Committee on Causality Assessment, whose task is to evaluate the role of immunization as the cause of reactions reported either by IMPACT or through other sources (16). These systems are complimentary to one another and form a network of surveillance activities that ensure adequate safety monitoring of licensed vaccines in Canada. The recent rotavirus vaccine story illustrates the usefulness of these activities, and should convince Health Canada that continued funding of IMPACT and of the other components of the vaccine safety monitoring program is of great importance to the health of Canadians.

\section{REFERENCES}

1. Rotavirus vaccine for the prevention of rotavirus gastroenteritis among children. Recommendations of the Advisory Committee on Immunization Practices (ACIP). MMWR Morb Mortal Wkly Rep 1999;48(RR-2):1-23.

2. Midthun $\mathrm{K}$, Zapikian AZ. Rotavirus vaccines: an overview. Clin Microbiol Rev 1996;9:423-34.

3. Kilgore PE, Holman RC, Clarke MJ, Glass RI. Trends of diarrheal disease - associated mortality in US children, 1968 through 1991. JAMA 1995;274:1143-8.

4. Parashar UD, Holman RC, Clarke MJ, Bresee JS, Glass RI. Hospitalization associated with rotavirus diarrhea in the United States, 1993 through 1995: Surveillance based on the new ICD-9-CM rotavirus-specific diagnostic code. J Infect Dis 1998;177:13-7.

5. Glass RI, Kilgore PE. Holman RC, et al. The epidemiology of rotavirus diarrhea in the United States: surveillance and 
estimates of disease burden. J Infect Dis 1996;174(Suppl 1):S5-11.

6. Rennels MB, Glass RI, Dennehy PH, et al. Safety and efficacy of high-dose rhesus-human reassortant rotavirus vaccines - report of a national multicenter trial. United States Rotavirus Vaccine Efficacy Group. Pediatrics 1996;97:7-13.

7. Perez-Schael I, Guntiñas MJ, Perez M, et al. Efficacy of the rhesus rotavirus-based quadrivalent vaccine in infants and young children in Venezuela. New Engl J Med 1997;337:1181-7.

8. Joensuu J, Koskenniemi E, Pang XL, Vesikari T. Randomised placebo-controlled trial of rhesus-human reassortant rotavirus vaccine for prevention of severe rotavirus gastroenteritis. Lancet 1997;350:1205-9.

9. Bernstein DI, Glass RI, Rogers G, Davidson BL, Sack DA. Evaluation of rhesus rotavirus monovalent and tetravalent reassortant vaccine in US children. US Rotavirus Vaccine Efficacy Group. JAMA 1995;273:1191-6.

10. Santosham M, Moulton LH, Reid R, et al. Efficacy and safety of high-dose rhesus-human reassortant rotavirus vaccine in native American population. J Pediatr 1997;131:632-8.
11. Prevention of rotavirus disease: guidelines for use of rotavirus vaccine. American Academy of Pediatrics. Pediatrics 1998; 102:1483-91.

12. Intussusception among recipients of rotavirus vaccine United States, 1998-1999. MMWR Morb Mortal Wkly Rep 1999;48:577-81.

13. Withdrawal of rotavirus vaccine recommendations. MMWR Morb Mortal Wkly Rep 1999;48:1007.

14. Scheifele D, Morris R, Halperin S, et al. IMPACT monitoring network: a better mousetrap. Can J Infect Dis 1993;4:194-5.

15. Division of Immunization, Bureau of Infectious Diseases, Laboratory Centre for Disease Control, Health Canada. Canadian National Report on Immunization, 1998. Paediatr Child Health 1999;4(Suppl C):7C-36C.

16. Pless R, Duclos P, and the Advisory Committee on Causality Assessment. Reinforcing surveillance for vaccine-associated adverse events. The Advisory Committee on Causality Assessment. Paediatr Child Health $1996 ; 1: 13-6$ 


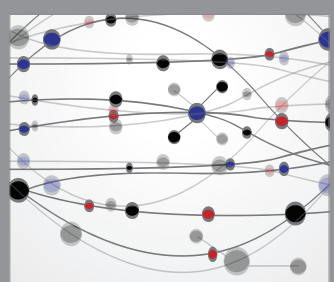

The Scientific World Journal
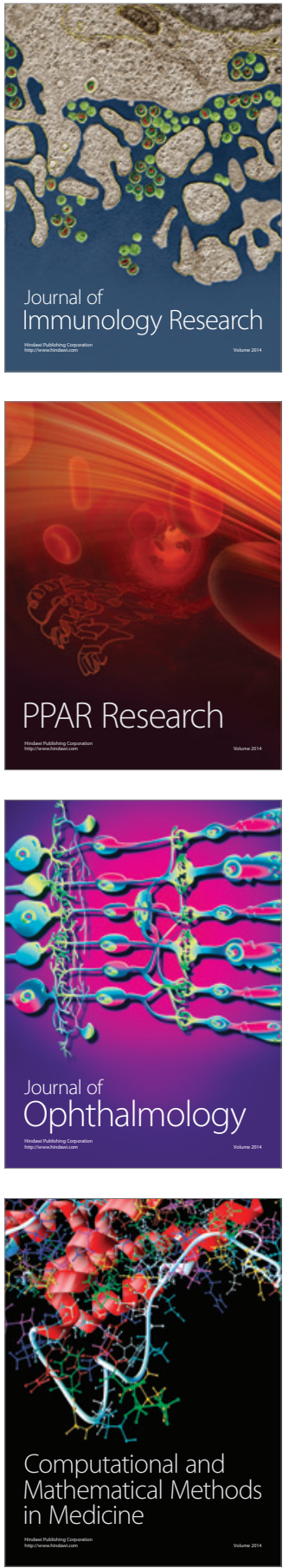

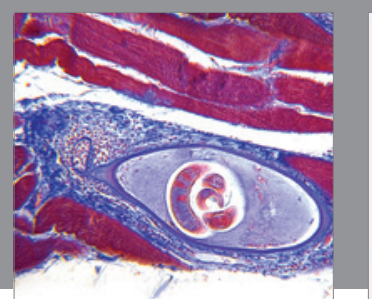

Gastroenterology Research and Practice

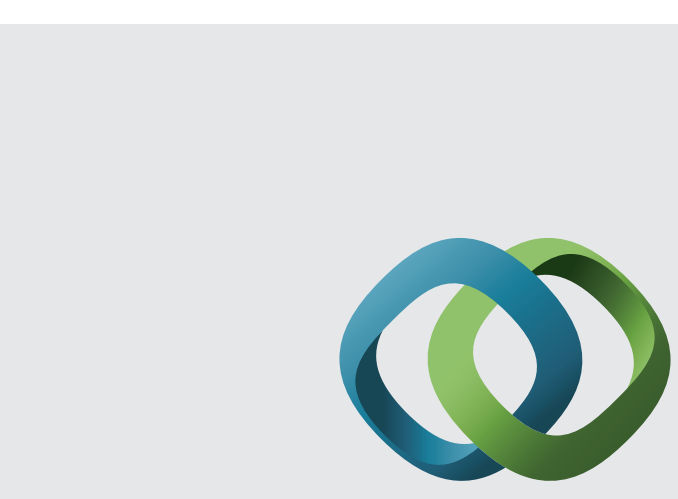

\section{Hindawi}

Submit your manuscripts at

http://www.hindawi.com
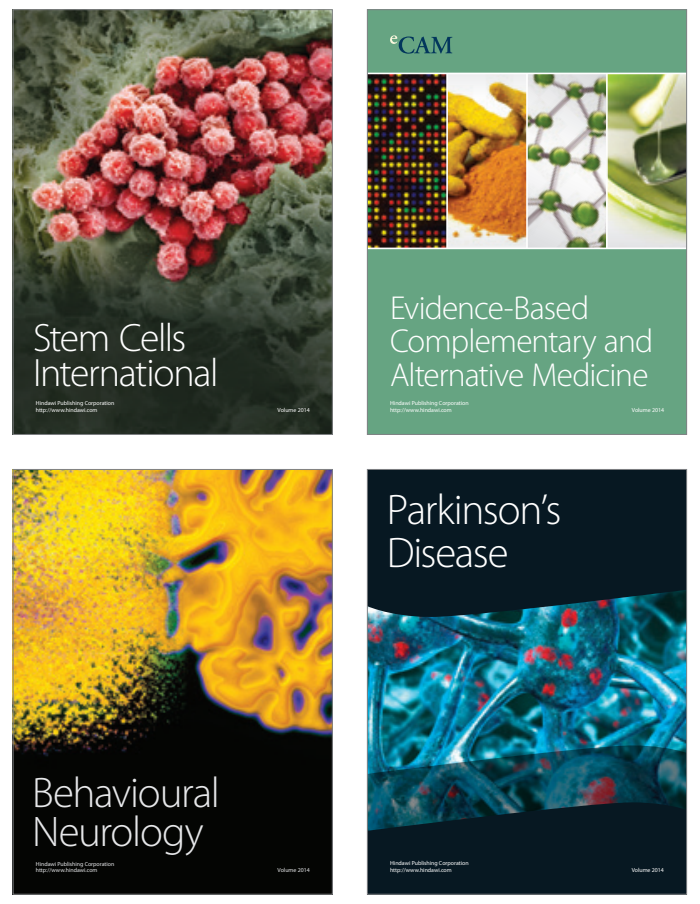
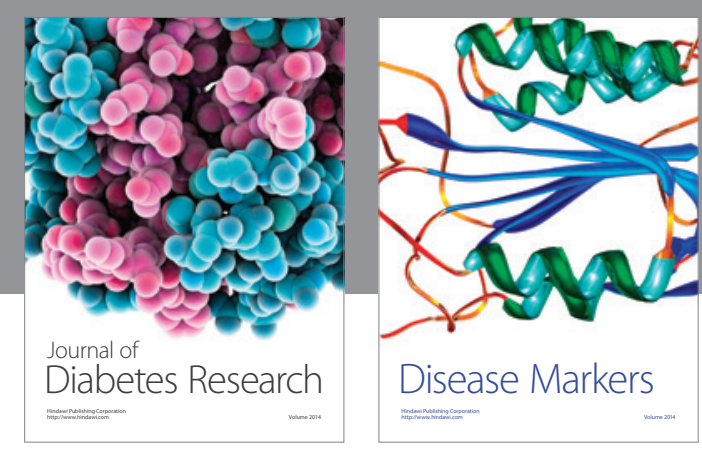

Disease Markers
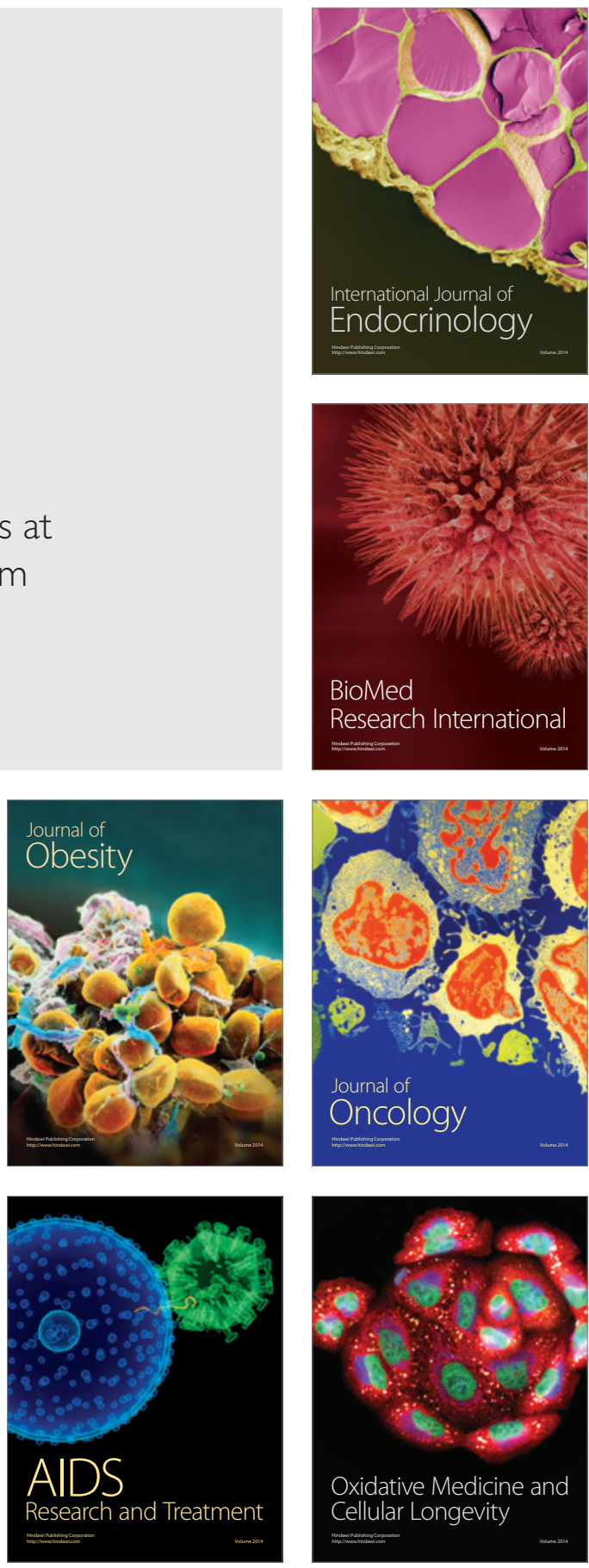